\title{
AVALIAÇÃO E VALIDAÇÃO DE FUNÇÕES DE AFILAMENTO PARA Mimosa scabrella Bentham EM POVOAMENTOS DA REGIÃO METROPOLITANA DE CURITIBA/PR
}

\author{
Daniel Queiroz*, Sebastião do Amaral Machado**, Afonso Figueiredo Filho***, \\ Júlio Eduardo Arce****, Henrique Soares Koehler***** \\ *Eng. Florestal, Mestrando em Eng. Florestal, UFPR - daniitapeva@bol.com.br \\ **Eng. Florestal, Dr., Depto. de Ciências Florestais, UFPR, Pesquisador do CNPq - sammac@floresta.ufpr.br \\ ***Eng. Florestal, Dr., Depto. de Ciências Exatas e Naturais, UNICENTRO - afig@floresta.ufpr.br \\ ****Eng. Florestal, Dr., Depto. de Ciências Florestais, UFPR - jarce@floresta.ufpr.br \\ *****Eng. Florestal, Dr., Depto. de Fitotecnia - koehler@ufpr.br \\ Recebido para publicação: 19/01/2006 - Aceito para publicação: 20/05/2006
}

\begin{abstract}
Resumo
Este estudo foi realizado com o propósito de avaliar a acurácia de três funções de afilamento para estimar diâmetros e volumes ao longo do fuste de Mimosa scabrella. A base de dados foi composta por 121 árvores cubadas pelo método de Hohenadl com dez seções e 53 árvores pelo método do deslocamento de água (xilômetro), obtidas na Região Metropolitana de Curitiba/PR. Os modelos testados foram o de Schöepfer (1966), o de Hradetzky (1976) e o de Kozak, Munro e Smith (1969). A acurácia dos modelos testados para as estimativas foi avaliada através de análise gráfica dos resíduos, coeficiente de determinação ajustado $\left(\mathrm{R}^{2}\right)$, erro padrão da estimativa (Syx\%) e análise gráfica dos perfis médios. Os volumes das 53 árvores xilometradas foram então estimados através da equação de maior acurácia, tanto para os dados agrupados por classe de idade como para o conjunto total. Os volumes estimados dessas 53 árvores foram comparados com seus respectivos volumes reais através da estatística qui-quadrado. Concluiu-se que o modelo de Hradetzky (1976) foi o melhor, tanto para estimativa dos diâmetros como dos volumes, em quase todas as classes de idade, e o melhor para o conjunto total dos dados. No teste da validação, verificou-se que as equações de afilamento de melhor desempenho para cada classe de idade e para o conjunto total dos dados resultaram em estimativas sem tendência de volumes e que, portanto, podem ser utilizadas para se fazer o sortimento.

Palavras-chave: Funções de afilamento; polinômio não-segmentados; validação de modelos.
\end{abstract}

\begin{abstract}
Evaluation and validation of taper functions for Mimosa scabrella Bentham in stands in the Curitiba Metropolitan Region. This study was accomplished with the objectives: to evaluate the acuracy of non segmented polynomial models to estimate diameters and volumes along the stem of Mimosa scabrella. The data base was composed by 174 trees of Mymosa scabrella, where 121 trees were cubed by the method of Hohenadl with ten sections and 53 trees by the method of water displacement (xilometer). All sample trees came from the Metropolitan Area of Curitiba/Paraná. The tested models were those from Schöepfer (1966), Hradetzky (1976) and Kozak, Munro and Smith (1969). The acuracy of the tested models for the estimates was evaluated through: graphic analysis of residuals, adjusted $\mathrm{R}^{2}$, standard error of estimate in percentage (Syx\%) and graphic analysis of average profiles, just used to verify if the fittings were satisfactory in a general way. The volumes of the 53 xilometered trees were then estimated through the equation of better performance, as by age class, as for the total of trees. The estimated volumes of these 53 trees were compared with their respective observed volumes through the $\chi^{2}$ statistic. After the analysis of results it was concluded that the model from Hradetzky (1976) was the best, as for estimating diameters, as for estimating volumes, in almost all age classes. It was also the best for the total data set. The validation test proved that taper equations of best performance for diameter estimation along the stem were also the best for volume estimation. Therefore they can be used to do the assortment.
\end{abstract}

Keywords: Taper functions; non segmented polynomial model; validation of models. 


\section{INTRODUÇÃO}

Mimosa scabrella Bentham (bracatinga) é uma espécie da família Leguminosae e da subfamília Mimosoideae. Sua área de ocorrência natural situa-se nos locais de climas mais frios do Brasil, sendo encontrada desde o sul de Minas Gerais até as bordas da Serra Geral, no Rio Grande do Sul (PEDROSO; MATTOS, 1987).

A bracatinga vem sendo cultivada no sul do país desde o início do século XX. No Paraná, esse cultivo se intensificou devido à propaganda que visava a atender a demanda energética (BARTOSZECK, 2000).

O sistema de manejo dessa espécie é realizado de modo predominante em pequenas propriedades agrícolas, caracterizado pela regeneração natural, via sementes, e induzido pela queima de restos da exploração florestal anterior. No primeiro ano, os bracatingais são consorciados com culturas de ciclo curto, principalmente milho e feijão. Após o cultivo agrícola inicial, como regra, não são realizados raleios. No bracatingal assim conduzido, estabelece-se forte competição e há mortalidade acentuada até a idade de 4 a 5 anos, quando a densidade torna-se mais estável. O número de plantas por hectare na idade de corte (6 a 8 anos) fica relativamente constante (CARPANEZZI et al., 1988).

O mais importante uso atual da madeira é para energia como lenha e, secundariamente, como carvão. A madeira roliça é muito procurada também para escoras na construção civil. Peças finas, retiradas aos dois anos, são usadas como varas de horta, tendo pouca durabilidade, motivo pelo qual os produtores dão preferência à taquara, quando disponível (CARPANEZZI et al., 1988). Existem outros usos dessa espécie, dentre eles a recuperação de áreas degradadas, forragem, fabricação de compensados, laminados, aglomerados e moirões.

Tonon (1998) já chamava a atenção para a possibilidade de melhoria técnica por outros manejos para a condução dos povoamentos. Uma justificativa para o propósito desse estudo seriam usos alternativos da espécie, como, por exemplo, a madeira serrada, que implicaria um manejo para a produção de árvores com características desejáveis.

No Sul do país, principalmente na Região Metropolitana de Curitiba, a bracatinga vem sendo substituída por espécies exóticas, com destaque para o Pinus sp, devido a razões de mercado e principalmente porque as grandes empresas florestais têm dado incentivo aos pequenos produtores com o fomento florestal.

Visando uma valorização e otimização do uso da bracatinga, o presente estudo teve como objetivo dar-lhe um uso mais nobre, pela técnica de sortimento. Para que se faça o uso dessa técnica, destaca-se o emprego de funções de afilamento, que se caracterizam pela sua grande flexibilidade e dinâmica, possibilitando representar o perfil do tronco das árvores, a partir do qual pode-se estimar o número de toras vinculadas às dimensões mínimas de cada produto a ser obtido da floresta, o que permite um planejamento da produção e da logística de transporte e comercialização, em função do número de toras destinadas a cada produto (ASSIS, 2000).

No Brasil, o foco dos estudos em que se empregam funções de afilamento ainda se restringe a espécies exóticas, entre elas o Pinus sp e o Eucalyptus sp. Considerando o exposto e a grande diversidade de espécies existentes, este trabalho contribui para que se conduzam trabalhos para outras espécies, como as nativas.

Essas funções de afilamento, quando comparadas com as funções de volume, possuem precisão equivalente, mas possuem muito mais vantagens, na medida em que se consegue estimar volume de qualquer porção da árvore devido a sua alta flexibilidade, possibilitando assim maior amplitude de informações geradas.

De acordo com Ahrens e Holbert (1981), uma função de afilamento é uma descrição matemática do perfil longitudinal de um tronco. Assumindo-se que a seção transversal seja circular em qualquer ponto ao longo do tronco, o seu volume pode ser obtido por integração daquela função. Assim, em termos geométricos, o tronco é tratado como um sólido de revolução. Uma vez definido um modelo matemático para o afilamento, pode-se determinar o volume de madeira entre quaisquer pontos ao longo do tronco. Dessa maneira, a natureza do algoritmo viabiliza a obtenção das estimativas de volume necessárias à multiplicidade de usos da madeira.

O presente trabalho teve como objetivos:

- Ajustar e comparar diferentes funções de afilamento para o total das árvores amostradas e por classe de idade para a Mimosa scabrella, visando à valorização dessa espécie florestal. 
- Verificar a acurácia dos modelos polinomiais não segmentados para estimar volumes comerciais e totais, e diâmetros ao longo do fuste.

- Verificar se o melhor modelo para estimar os diâmetros é também o melhor para estimar os volumes.

- Analisar o comportamento do fuste com o aumento da idade.

- Validar as equações de afilamento com os respectivos volumes reais obtidos com a técnica de deslocamento de água (xilômetro).

\section{MATERIAL E MÉTODOS}

\section{Localização e caracterização da área de estudo}

Para o desenvolvimento desta pesquisa, foram utilizados dados de povoamentos de bracatinga localizados na Região Metropolitana de Curitiba, estado do Paraná, na qual se incluem 14 municípios, abrangendo uma área de 876.300 ha. Esses dados foram, na maioria, coletados na porção norte da região, onde há uma maior ocorrência da espécie, incluindo os municípios de Almirante Tamandaré, Bocaiúva do Sul, Campina Grande do Sul, Campo Magro, Colombo, Itaperuçu, Rio Branco e Tunas. Esses municípios situam-se entre as latitudes $25^{\circ} 11^{\prime}$ e $25^{\circ} 49^{\prime}$ S e entre as longitudes $49^{\circ} 05^{\prime}$ e $49^{\circ} 43^{\prime}$ W.

Segundo o sistema de classificação de Köppen, o clima da região é do tipo $C f b$, que corresponde ao clima temperado propriamente dito, mesotérmico úmido sem estação seca definida, com quatro estações bem definidas, com verões quentes e chuvosos e invernos frios com períodos secos eventuais. A temperatura média do mês mais frio é inferior a $18{ }^{\circ} \mathrm{C}$ e a do mês mais quente fica abaixo de $22{ }^{\circ} \mathrm{C}$. A temperatura média anual se apresenta em torno de $17{ }^{\circ} \mathrm{C}$, com mínimas de $12{ }^{\circ} \mathrm{C}$ e máximas de $23{ }^{\circ} \mathrm{C}$. A precipitação média anual situa-se entre 1.300 e $2.500 \mathrm{~mm}$, com leve diminuição no inverno, não ocorrendo déficits hídricos (MAACK, 1981; IAPAR, 1994).

\section{Volumetria}

Foram cubadas 174 árvores de Mimosa scabrella Bentham com idades variando de 6 a 19 anos.

A cubagem de 121 árvores foi feita pelo método relativo, tomando-se as medidas relativas dos diâmetros a $0 \%, 5 \%, 15 \%, 25 \%, 35 \%, 45 \%, 55 \%, 65 \%, 75 \%, 85 \%, 95 \%$ e $100 \%$ da altura total do fuste, não considerando a altura do toco. Para definir a altura de cada fuste, foi considerada a inversão morfológica do tronco mais característico, não sendo estabelecido um diâmetro mínimo.

O cálculo dos volumes das seções foi feito utilizando-se a fórmula de Hohenadl:

$$
\mathrm{v}=\frac{\mathrm{hf}}{10}\left[\mathrm{~g}_{0,05^{+}} \mathrm{g}_{0,15^{+}} \mathrm{g}_{0,25} \mathrm{~g}_{0,35^{+}} \mathrm{g}_{0,45^{+}}+\mathrm{g}_{0,55^{+}} \mathrm{g}_{0,65}+\mathrm{g}_{0,75^{+}} \mathrm{g}_{0,85^{+}} \mathrm{g}_{0,95}\right]
$$

Sendo: $\mathrm{v}=$ volume de árvore considerada $\left(\mathrm{m}^{3}\right)$;

$\mathrm{hf}=$ altura do fuste $(\mathrm{m})$

$\mathrm{g}_{0, \mathrm{i}}=$ área seccional $\left(\mathrm{m}^{2}\right)$ com diâmetros tomados a $5,15,25,35,45,55,65,75,85,95 \%$ da altura do fuste da árvore.

Os volumes das outras 53 árvores foram obtidos pela técnica de deslocamento de água xilômetro construído por Machado e Nadolny (1991). O xilômetro determina o volume verdadeiro de corpos de qualquer forma, valendo-se do princípio de deslocamento de um líquido (água).

As 121 árvores cubadas pelo método de Hohenadl foram distribuídas em 4 classes de idade, com o mínimo de 16 árvores em cada classe, conforme mostrado na tabela 1.

Tabela 1. Distribuição das árvores cubadas em diferentes classes de idades.

Table 1. Distribution of the cubed trees in different age classes.

\begin{tabular}{ccc}
\hline Número da classe & Classe de idade (anos) & Freqüência \\
\hline 1 & $6-9$ & 24 \\
2 & $10-12$ & 55 \\
3 & $13-15$ & 26 \\
4 & $16-19$ & 16 \\
\hline Total & - & 121 \\
\hline
\end{tabular}


Os ajustes dos modelos foram feitos considerando-se os diâmetros a 1,3 m do solo (DAP), com casca, relacionados aos diâmetros comerciais com casca e as alturas relativas: $0,5,15,25,35,45,55,65$, $75,85,95$ e $100 \%$ da altura do fuste da árvore.

\section{Funções de afilamento não-segmentadas testadas}

$\mathrm{O}$ ajuste dos modelos não-segmentados foi feito para cada classe de idade e também para o conjunto total dos dados (não agrupados).

Os modelos não-segmentados testados são descritos a seguir:

a) Polinômio de Quinto Grau - Modelo 1

O polinômio de quinto grau foi proposto primeiramente por Schöepfer (1966).

$$
\frac{\mathrm{di}}{\mathrm{d}_{1,3}}=\beta_{0}+\beta_{1}\left(\frac{\mathrm{h}_{\mathrm{i}}}{\mathrm{hf}}\right)+\beta_{2}\left(\frac{\mathrm{h}_{\mathrm{i}}}{\mathrm{hf}}\right)^{2}+\beta_{3}\left(\frac{\mathrm{h}_{\mathrm{i}}}{\mathrm{hf}}\right)^{3}+\beta_{4}\left(\frac{\mathrm{h}_{\mathrm{i}}}{\mathrm{hf}}\right)^{4}+\beta_{5}\left(\frac{\mathrm{h}_{\mathrm{i}}}{\mathrm{hf}}\right)^{5}+\mathrm{e}_{\mathrm{i}}
$$

Sendo: $\beta_{\mathrm{i}}=$ parâmetros a serem estimados;

$\mathrm{d}_{\mathrm{i}}=$ diâmetro tomado às diferentes alturas $\mathrm{h}_{\mathrm{i}}$ ao longo do fuste;

$\mathrm{d}_{1,3}=$ diâmetro a $1,3 \mathrm{~m}$ de altura (DAP);

$\mathrm{hf}=$ altura do fuste $(\mathrm{m})$;

$\mathrm{h}_{\mathrm{i}}=$ altura i (m) de um referido diâmetro;

$\mathrm{e}_{\mathrm{i}}=$ erro de estimativa.

b) Polinômio de Potências Fracionárias (HRADETZKY, 1976) - Modelo 2

Esse modelo diferencia-se do Polinômio de Quinto Grau por apresentar um conjunto de potências fracionárias como tentativa de melhor representar o afilamento da árvore, principalmente nos dois extremos.

$$
\frac{\mathrm{di}}{\mathrm{d}_{1,3}}=\beta_{0}+\beta_{1}\left(\frac{\mathrm{h}_{\mathrm{i}}}{\mathrm{hf}}\right)^{\mathrm{p} 1}+\beta_{2}\left(\frac{\mathrm{h}_{\mathrm{i}}}{\mathrm{hf}}\right)^{\mathrm{p} 2}+\ldots+\beta_{5}\left(\frac{\mathrm{h}_{\mathrm{i}}}{\mathrm{hf}}\right)^{\mathrm{pn}}+\mathrm{e}_{\mathrm{i}}
$$

Sendo: $d_{i}, d_{1,3}, h_{i}$, hf, $\beta_{i}$ e e $e_{i}=$ já definidos anteriormente;

$\mathrm{p}_{\mathrm{i}}=$ expoentes variando entre 0,00005 e 95 .

c) Polinômio de Segundo Grau (KOZAK et. al. 1969) - Modelo 3

A fórmula geral do Polinômio de Segundo Grau é:

$$
\frac{\mathrm{d}_{\mathrm{i}}}{\mathrm{d}_{1,3}}=\sqrt{\beta_{0}+\beta_{1}\left(\frac{\mathrm{h}_{\mathrm{i}}}{\mathrm{hf}}\right)+\beta_{2}\left(\frac{\mathrm{h}_{\mathrm{i}}}{\mathrm{hf}}\right)^{2}+\mathrm{e}_{\mathrm{i}}}
$$

Sendo: $d_{i}, d_{1,3}, h_{i}$, hf, $\beta_{i}$ e $e_{i}$ já definidos anteriormente.

Integrando esses modelos, chega-se à expressão que propicia a obtenção dos sortimentos ou volumes comerciais correspondentes a qualquer porção da árvore, além do volume total.

\section{Ajustes dos modelos}

Para o ajuste dos modelos de afilamento, foi utilizado o programa Statgraphics plus 2.1, visando à obtenção dos coeficientes de regressão, das estatísticas de comparação e análise de resíduos. Para o ajuste do modelo de Potências Fracionárias (HRADETZKY, 1976), utilizou-se o método Backward, o qual consiste em eliminar todas as variáveis que não atingem o grau de significância determinado para o teste. Houve uma combinação de potências que foi desde 0,00005/0,00006 até 95 .

As informações obtidas através desse processamento foram organizadas a fim de se realizarem as análises estatísticas. 


\section{Acurácia dos modelos de afilamento}

Para testar os três modelos ajustados, foram utilizadas as estatísticas análise gráfica dos resíduos, coeficiente de determinação corrigido $\left(\mathrm{R}^{2}\right)$, erro padrão da estimativa $($ Syx $\%)$ e análise gráfica dos perfis médios. Elas foram utilizadas apenas para verificar se os modelos possuem ajustes satisfatórios de maneira geral. Complementando o teste de acurácia dos modelos, foram utilizadas estatísticas utilizadas por Figueiredo Filho, Borders e Hitch (1996), Ferreira (1999), Assis (2000) e Ferreira (2004). Tais estatísticas permitem uma análise mais detalhada do desempenho das estimativas ao longo de todo o fuste, uma vez que são calculadas para cada altura relativa onde são tomados os diâmetros por ocasião da cubagem rigorosa. Foram avaliadas as variáveis diâmetros estimados em cada posição de medição (alturas relativas) e os volumes parciais correspondentes ao volume calculado, a partir do método de Hohenadl, além do volume total. A tabela 2 apresenta as estatísticas que foram utilizadas no teste da acurácia.

Tabela 2. Estatísticas utilizadas para avaliação das estimativas dos diâmetros e volumes comerciais.

Table 2. Utilized statistics for evaluation of the estimates of diameters and commercial volumes.

\begin{tabular}{|c|c|c|c|}
\hline & & Diâmetros & Volumes \\
\hline 1) & Desvio (D) & $\left(\sum\right.$ Diff $\left._{i}\right) / \mathrm{N}$ & $\left(\sum\right.$ Diff $\left._{\mathrm{i}}\right) / \mathrm{N}$ \\
\hline 2) & Desvio Padrão das Diferenças (SD) & {$\left[\sum\left(\operatorname{Diff}-\mathrm{d}_{\mathrm{i}}\right)^{2} /(\mathrm{N}-1)\right]^{0,5}$} & {$\left[\sum\left(\text { Diff }-\mathrm{v}_{\mathrm{i}}\right)^{2} /(\mathrm{N}-1)\right]^{0,5}$} \\
\hline 3) & $\begin{array}{l}\text { Soma do Quadrado do Resíduo } \\
\text { Relativo (SQRR) }\end{array}$ & $\sum\left(\text { Diff }_{i} / d_{i}\right)^{2}$ & $\sum\left(\operatorname{Diff}_{\mathrm{i}} / \mathrm{v}_{\mathrm{i}}\right)^{2}$ \\
\hline 4) & Porcentagem dos Resíduos (RP) & $\sum\left(\right.$ Diff $\left._{\mathrm{i}} / \mathrm{d}_{\mathrm{i}}\right) * 100 / \mathrm{N}$ & $\sum\left(\right.$ Diff $\left._{\mathrm{i}} / \mathrm{v}_{\mathrm{i}}\right) * 100 / \mathrm{N}$ \\
\hline
\end{tabular}

Sendo: $\operatorname{Diff}_{\mathrm{i}}=\left(\mathrm{v}_{\mathrm{i}}-\hat{\mathrm{v} i}\right)$ ou $\left(\mathrm{d}_{\mathrm{i}}-\hat{\mathrm{di}}\right)$ é o desvio dos volumes ou dos diâmetros;

$\mathrm{v}_{\mathrm{i}}=$ volume total ou comercial até o diâmetro i da árvore;

$\hat{\mathrm{v}} \mathrm{i}=$ volume total ou comercial estimado;

$\mathrm{d}_{\mathrm{i}}=$ diâmetro da árvore na posição i do fuste;

$\mathrm{N}=$ número de árvores.

A partir dessas estatísticas, foi elaborado um ranking para expressar o desempenho dos modelos testados para estimar os diâmetros ao longo do fuste, e os volumes totais e parciais das árvores em estudo. Esse ranking foi feito para cada classe de idade, e também para o conjunto total dos dados, para as variáveis diâmetro e volume. Considerando-se cada uma das estatísticas testadas, conforme a tabela 2 , foi atribuída nota 1 para o modelo que apresentou melhor acurácia em cada posição relativa da medição, e assim sucessivamente até a nota 3 para a equação que propiciou as estatísticas menos acuradas. Equações com mesmo valor das estatísticas receberam as mesmas notas.

\section{Validação das funções de afilamento}

As árvores cubadas com a técnica do deslocamento de água (xilômetro) foram utilizadas para o processo de validação das equações de afilamento ajustadas. Esse processo consistiu em estimar os volumes dessas árvores pela função de afilamento de melhor acurácia e compará-las com seus respectivos volumes xilometrados pelo teste de qui-quadrado $\left(\chi^{2}\right)$.

$$
\chi_{\text {CALCULAD }}^{2}=\sum \frac{\left(\mathrm{y}_{\mathrm{i}}-\hat{\mathrm{y}}_{\mathrm{i}}\right)^{2}}{\hat{\mathrm{y}}_{\mathrm{i}}}
$$

Sendo: $\mathrm{y}_{\mathrm{i}}=$ volume real (xilômetro);

$\hat{y}_{\mathrm{i}}=$ volume estimado (função de afilamento). 
Para a equação de regressão utilizando o conjunto total de dados, utilizaram-se todas as árvores cubadas com o xilômetro, e para as equações ajustadas por classe de idade, utilizaram-se apenas as árvores pertencentes a cada classe (Tabela 3 ).

A amostra para a validação deve ser independente dos dados utilizados no ajuste, cobrindo todas as condições de idade, sítios e de manejo (PRODAN, 1997). Segundo Snee citado por Prodan et al. (1997), depois do desenvolvimento de um modelo de regressão deve ser realizado o controle da validação e qualidade de suas predições, de modo que se tenha a confiança do modelo desenvolvido.

Tabela 3. Freqüência de árvores cubadas pelos métodos de Hohenadl e xilômetro, nas diferentes classes de idade.

Table 3. Distribution of the cubed trees by the Hohenadl and xilometer methods at the different age classes.

\begin{tabular}{cccc}
\hline Número da classe & Classe de idade (anos) & Método Hohenadl & Método xilômetro \\
\hline 1 & $6-9$ & 24 & 22 \\
2 & $10-12$ & 55 & 23 \\
3 & $13-15$ & 26 & 5 \\
4 & $16-19$ & 16 & 3 \\
\hline Total & - & 121 & 53 \\
\hline
\end{tabular}

\section{RESULTADOS E DISCUSSÃO}

\section{Ajustes dos modelos}

Os parâmetros estimados para os três modelos testados por classe de idade e para o conjunto total dos dados são apresentados na tabela 4, com os respectivos Coeficientes de Determinação Corrigido $\left(\mathrm{R}^{2}\right)$ e Erros Padrões da Estimativa em percentagem (Syx\%). Essas estatísticas retratam valores médios, não fornecendo um bom indicativo do comportamento ao longo do fuste. Esses testes foram complementados pelas estatísticas apresentadas na tabela 2, permitindo assim uma análise mais detalhada.

Em geral, os modelos testados apresentaram Coeficiente de Determinação $\left(\mathrm{R}^{2}\right)$ inferior a $0,93 \mathrm{e}$ Erro Padrão da Estimativa (Syx\%) entre 10 e 15\%. Apenas na classe de idade 6 a 9 anos e para o conjunto total de árvores o $\mathrm{R}^{2}$ foi maior que 0,9 para todos os modelos testados. O modelo de Kozak et al. (1969) mostrou os menores $\mathrm{R}^{2}$ em todas as classes de idade, quando comparado com os outros dois modelos testados.

\section{Acurácia dos modelos não-segmentados para a estimativa dos diâmetros}

Como a avaliação geral dos modelos foi feita levando-se em conta não somente as estatísticas $\mathrm{R}^{2}$ ajustado (\%) e Syx(\%), mas também a análise gráfica dos resíduos, pode-se observar que nem sempre o modelo que apresentou a melhor pontuação, conforme será explicado a seguir, deixa de apresentar tendenciosidade ao longo da linha média, indicando que a pontuação não é o único fator decisivo na escolha de um modelo.

Todos os modelos apresentaram alguma tendenciosidade ao longo da linha estimativa (Figura 1). Os gráficos dos resíduos foram feitos também para cada classe de idade, possibilitando assim uma avaliação minuciosa do comportamento das estimativas ao longo da linha média. Todos os modelos testados nas diferentes classes de idade apresentaram tendenciosidade ao longo da linha média. Os maiores erros geralmente ocorreram na porção inferior do fuste, onde a variação dos diâmetros no processo de cubagem foram muito bruscas.

Na tabela 5 são apresentadas as estatísticas: Desvio (D), Soma de Quadrados dos Resíduos Relativos (SQRR), Desvio Padrão das Diferenças (SD) e Resíduo Percentual (RP) referentes aos resultados obtidos pelos três modelos polinomiais não-segmentados para o ajuste total de dados. Esses cálculos também foram feitos para cada classe de idade, como mostrado na tabela 6 .

A partir dessas estatísticas, foi feita uma classificação, na qual o modelo testado que apresentou o menor valor no somatório foi o modelo mais acurado para estimar o diâmetro naquela posição do fuste. Exemplificando, o modelo de Hradetzky, entre os três modelos analisados, foi o que apresentou o menor 
somatório na posição da altura relativa $0 \%$ para estimativa dos diâmetros (com a nota 6), apresentando o primeiro lugar para a estatística $\mathrm{D}$, primeiro para a estatística SQRR, segundo para a estatística SD e primeiro para estatística RP, conforme observado na tabela 5 . Na segunda posição, com a nota 8 , ficou o modelo de $5^{\circ}$ Grau, e em último lugar o modelo de Kozak et al. (1969), com a nota 10. Em quase todas as posições da altura relativa, o modelo de Hradetzky (1976) se destacou em primeiro lugar, sendo que apenas na posição de $35 \%$ o modelo de $5^{\circ}$ Grau obteve o primeiro lugar, com a nota 6 , seguido pelo modelo de Hradetzky, com 7, e pelo de Kozak, com 11. Já na posição de 95\%, houve empate em primeiro lugar entre o modelo de $5^{\circ}$ Grau e o modelo de Hradetzky (1969), com nota 7 cada um deles, seguidos do modelo de Kozak, com 10. Então, na altura relativa de $95 \%$, os modelos de Hradetzky e o de $5^{\circ}$ Grau foram os que apresentaram estimativa mais acurada para o diâmetro. Dessa mesma forma foi possível determinar o modelo mais acurado para a estimativa do diâmetro nas diferentes posições relativas da altura do fuste nas 4 classes de idade.

Tabela 4. Parâmetros estimados e medidas de precisão para os modelos ajustados e respectivas medidas de precisão, para as quatro classes de idade e para o conjunto total dos dados.

Table 4. Estimated parameters and mensures of precision for the fitted models by age classes and for the whole data set.

\begin{tabular}{ccccccccc}
\hline & \multicolumn{8}{c}{ Modelo do Quinto Grau - Modelo 1 } \\
\hline \multirow{2}{*}{$\begin{array}{c}\text { Classe idade } \\
\text { (anos) }\end{array}$} & $\hat{\beta}_{0}$ & $\hat{\beta}_{1}$ & $\hat{\beta}_{2}$ & $\hat{\beta}_{3}$ & $\hat{\beta}_{4}$ & $\hat{\beta}_{5}$ & $\mathrm{R}^{2}$ & \multirow{2}{*}{$\mathrm{Syx} \%(\hat{\mathrm{di}})$} \\
\hline $6-9$ & 1,18136 & $-2,1841$ & 7,20916 & $-13,2895$ & 11,213 & $-3,56757$ & 0,93901 & 10,7046 \\
$10-12$ & 1,25787 & $-3,74865$ & 16,2077 & $-33,3579$ & 30,388 & $-10,1582$ & 0,90496 & 11,7342 \\
$13-15$ & 1,19409 & $-2,41612$ & 8,75301 & $-16,4941$ & 14,1881 & $-4,54168$ & 0,89270 & 11,5992 \\
$16-19$ & 1,20971 & $-2,75841$ & 10,4931 & $-21,0139$ & 19,187 & $-6,42512$ & 0,76225 & 11,9915 \\
Total & 1,22262 & $-3,02105$ & 12,0654 & $-24,1215$ & 21,6226 & $-7,15046$ & 0,92437 & 12,6160 \\
\hline
\end{tabular}

\begin{tabular}{|c|c|c|c|c|c|c|c|}
\hline \multirow{3}{*}{$\begin{array}{l}\text { Classe idade } \\
\text { (anos) }\end{array}$} & \multicolumn{7}{|c|}{ Modelo de Potências Fracionárias - Modelo 2} \\
\hline & & & $\overline{\text { Paráá }}$ & etros estim & & & \\
\hline & $\hat{\beta}_{0}$ & $\hat{\beta}_{1}$ & $\hat{\beta}_{2}$ & $\hat{\beta}_{3}$ & $\hat{\beta}_{4}$ & $\mathrm{R}^{2}$ & Syx\%( $\hat{d} \mathrm{i})$ \\
\hline $\begin{array}{c}6-9 \\
\text { Potências }\end{array}$ & 1,1913 & $\begin{array}{c}-0,067119 \\
0,00005\end{array}$ & $\begin{array}{c}-0,50608 \\
0,7\end{array}$ & $\begin{array}{c}-0,057774 \\
5\end{array}$ & & 0,93966 & 10,6474 \\
\hline $\begin{array}{c}10-12 \\
\text { Potências }\end{array}$ & 1,28259 & $\begin{array}{c}-0,125363 \\
0,00005\end{array}$ & $\begin{array}{c}-0,438911 \\
0,5\end{array}$ & $\begin{array}{c}-0,212281 \\
4\end{array}$ & $\begin{array}{c}0,0958567 \\
18\end{array}$ & 0,90864 & 11,5051 \\
\hline $\begin{array}{c}13-15 \\
\text { Potências }\end{array}$ & 1,19665 & $\begin{array}{c}-0,505281 \\
0,5\end{array}$ & & & & 0,89470 & 11,4904 \\
\hline $\begin{array}{c}16-19 \\
\text { Potências }\end{array}$ & 1,23251 & $\begin{array}{c}-0,549377 \\
0,4\end{array}$ & & & & 0,76648 & 11,8844 \\
\hline $\begin{array}{c}\text { Total } \\
\text { Potências }\end{array}$ & 1,24109 & $\begin{array}{c}-0,07589 \\
0,00005\end{array}$ & $\begin{array}{c}-0,459412 \\
0,5\end{array}$ & $\begin{array}{c}-0,145145 \\
4\end{array}$ & $\begin{array}{c}0,0654363 \\
17\end{array}$ & 0,92603 & 12,4767 \\
\hline
\end{tabular}

\begin{tabular}{cccccc}
\hline & \multicolumn{5}{c}{ Modelo de Kozak et al. (1969) - Modelo 3 } \\
\hline Classe idade & \multicolumn{5}{c}{ Parâmetros estimados } \\
\cline { 2 - 6 } (anos) & $\hat{\beta}_{0}$ & $\hat{\beta}_{1}$ & $\hat{\beta}_{2}$ & $\mathrm{R}^{2}$ & Syx\%( $\hat{\mathrm{d}} \mathrm{i})$ \\
\hline $6-9$ & 1,29196 & $-1,67533$ & 0,743909 & 0,93072 & 11,4091 \\
$10-12$ & 1,39729 & $-2,00489$ & 1,01853 & 0,88370 & 12,9807 \\
$13-15$ & 1,31091 & $-1,66001$ & 0,869279 & 0,88048 & 12,2420 \\
$16-19$ & 1,3409 & $-1,99327$ & 1,19017 & 0,74492 & 12,4208 \\
Total & 1,35038 & $-1,86388$ & 0,954686 & 0,91545 & 13,3395 \\
\hline
\end{tabular}


QUINTO GRAU

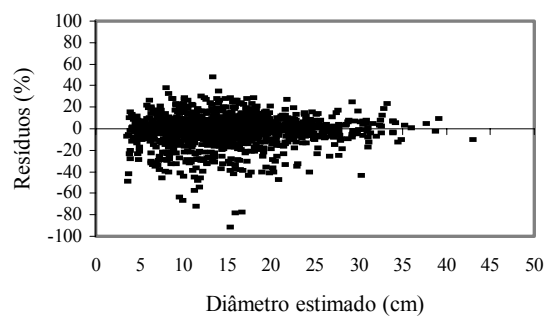

POTÊNCIAS FRACIONÁRIAS

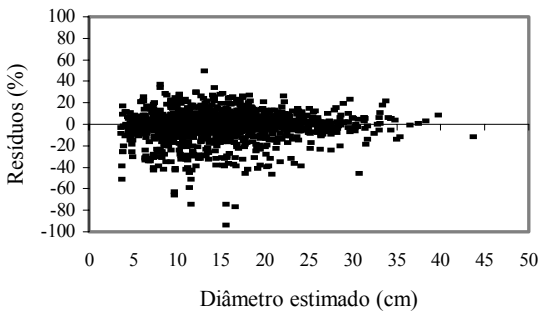

KOZAK et al. (1969)

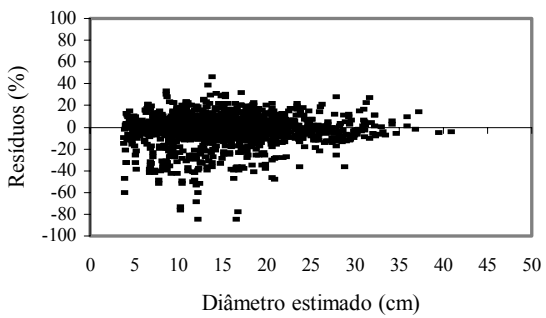

Figura 1. Distribuição gráfica dos resíduos em percentagem em função do diâmetro estimado para o conjunto total dos dados para os três modelos ajustados.

Figure 1. Residual graphic distribution in percent over estimated diameters for the whole data set for the three fitted models.

Em geral, em todas as classes de idade o modelo Hradetzky ocupou o primeiro lugar, excetuando-se apenas a classe de 6 a 9 anos, na qual o modelo de $5^{\circ}$ Grau foi o que ocupou o primeiro lugar.

Na tabela 7 são mostrados os resultados da classificação dos modelos, identificando o modelo mais acurado para estimar os diâmetros nas diferentes posições do fuste. A mesma tabela apresenta a porcentagem de vezes que cada modelo esteve em primeiro lugar nas posições. Dois modelos selecionados em uma mesma posição indicam que ambos obtiveram o mesmo número de pontos no processo de classificação. Quando se calcula a percentagem no caso de modelos empatados, considera-se o peso de 0,5 para cada um, de modo que o somatório seja de $100 \%$.

Para o conjunto total de árvores, o modelo de Potências Fracionárias foi o que estimou com maior acurácia os diâmetros em $88 \%$ dos casos. Apenas na posição relativa a $35 \%$ da altura do fuste o modelo do Polinômio de Quinto Grau obteve o primeiro lugar, e na posição de 95\% houve empate entre os modelos 1 e 2 .

Conforme mostrado na tabela 6, o modelo de Potências Fracionárias obteve os primeiros lugares nas classes de idade 10 a 12 anos, 13 a 15 anos e 16 a 19 anos, com $62 \%, 58 \%$ e $58 \%$, respectivamente. Apenas na classe de 6 a 9 anos o modelo do Polinômio de Quinto Grau obteve a melhor pontuação, com $54 \%$.

Na posição relativa de 5\% da altura do fuste, o modelo de Potências Fracionárias foi o melhor em todas as classes de idade, destacando-se também nas posições relativas de $0,5,15$ e $25 \%$, em que também esteve em primeiro, havendo alguns empates com o modelo do Polinômio de Quinto Grau.

Nas demais alturas relativas não houve uma tendência de indicação de um modelo mais eficiente, havendo uma mistura entre os modelos, principalmente o de Potências Fracionárias e o Polinômio do $5^{\circ}$ Grau; em poucos casos, o modelo de Kozak. 
Tabela 5. Estatísticas dos modelos polinomiais não-segmentados para a estimativa dos diâmetros ao longo do fuste das árvores de Mimosa scabrella, para o ajuste do conjunto total dos dados (não agrupados).

Table 5. Statistics of the fitted non segmented polynomial models for estimating diameters along the tree stem for the whole data set.

\begin{tabular}{|c|c|c|c|c|c|c|c|c|c|c|c|c|c|}
\hline \multirow[b]{2}{*}{ Modelo } & \multirow[b]{2}{*}{ Estat. } & \multicolumn{12}{|c|}{ Alturas relativas de medição (\%) } \\
\hline & & $\mathbf{0}$ & 5 & 15 & 25 & 35 & 45 & 55 & 65 & 75 & 85 & 95 & 100 \\
\hline \multirow{4}{*}{$5^{\circ} \mathrm{Grau}$} & $\mathrm{D}$ & 0,302 & $-0,631$ & 0,246 & 0,176 & $-0,011$ & $-0,158$ & $-0,192$ & 0,107 & 0,281 & $-0,064$ & $-0,080$ & 0,353 \\
\hline & SQRR & 2,108 & 0,605 & 0,328 & 0,358 & 0,561 & 0,720 & 1,064 & 1,435 & 1,814 & 2,912 & 5,151 & 5,821 \\
\hline & SD & 22,612 & 20,323 & 17,938 & 17,052 & 16,571 & 15,961 & 15,098 & 14,099 & 13,169 & 12,443 & 11,824 & 11,422 \\
\hline & $\mathrm{RP}$ & 1,511 & $-3,186$ & 1,632 & 1,128 & $-0,291$ & $-1,053$ & $-1,082$ & 0,833 & 1,885 & $-0,795$ & $-1,866$ & 1,338 \\
\hline \multirow{4}{*}{$\begin{array}{l}\text { Hradetzky } \\
(1976)\end{array}$} & $\mathrm{D}$ & $-0,016$ & $-0,004$ & $-0,052$ & $-0,046$ & 0,071 & 0,048 & $-0,104$ & $-0,007$ & 0,111 & $-0,015$ & 0,140 & 0,207 \\
\hline & SQRR & 2,019 & 0,516 & 0,286 & 0,334 & 0,566 & 0,728 & 1,065 & 1,403 & 1,726 & 2,930 & 5,319 & 5,643 \\
\hline & SD & 22,953 & 19,650 & 18,258 & 17,291 & 16,483 & 15,740 & 15,003 & 14,221 & 13,351 & 12,391 & 11,588 & 11,579 \\
\hline & $\mathrm{RP}$ & 0,000 & 0,127 & $-0,146$ & $-0,268$ & 0,239 & 0,338 & $-0,457$ & $-0,037$ & 0,494 & $-0,382$ & 0,133 & $-0,033$ \\
\hline \multirow{5}{*}{$\begin{array}{l}\text { Kozak et } \\
\text { al. (1969) }\end{array}$} & $\mathrm{D}$ & 1,346 & $-1,035$ & $-1,049$ & $-0,679$ & $-0,128$ & 0,245 & 0,372 & 0,555 & 0,495 & $-0,104$ & $-0,510$ & $-0,450$ \\
\hline & SQRR & 2,863 & 0,791 & 0,645 & 0,505 & 0,564 & 0,782 & 1,251 & 1,762 & 2,000 & 2,900 & 5,105 & 5,417 \\
\hline & SD & 21,492 & 20,757 & 19,329 & 17,970 & 16,696 & 15,528 & 14,492 & 13,618 & 12,939 & 12,487 & 12,286 & 12,284 \\
\hline & $\mathrm{RP}$ & 6,801 & $-5,210$ & $-5,680$ & $-4,035$ & $-1,040$ & 1,706 & 3,050 & 4,388 & 3,694 & $-1,143$ & $-5,556$ & $-5,773$ \\
\hline & \multicolumn{13}{|c|}{ Notas } \\
\hline \multicolumn{2}{|c|}{$5^{\circ} \mathrm{Grau}$} & 8 & 8 & 7 & 7 & 6 & 8 & 8 & 8 & 8 & 8 & 7 & 8 \\
\hline \multicolumn{2}{|c|}{ Hradetzky (1976) } & 6 & 4 & 5 & 5 & 7 & 6 & 6 & 6 & 6 & 6 & 7 & 6 \\
\hline \multicolumn{2}{|c|}{ Kozak et al. (1969) } & 10 & 12 & 12 & 12 & 11 & 10 & 10 & 10 & 10 & 10 & 10 & 10 \\
\hline
\end{tabular}

Tabela 6. Estatísticas dos modelos polinomiais não-segmentados para a estimativa dos diâmetros ao longo do fuste das árvores de Mimosa scabrella, na classe de idade 10 a 12 anos.

Table 6. Statistics of the fitted non segmented polynomial models for estimating diameters along the tree stem for the age class 10 to 12 years old.

\begin{tabular}{|c|c|c|c|c|c|c|c|c|c|c|c|c|c|}
\hline \multirow[b]{2}{*}{ Modelo } & \multirow[b]{2}{*}{ Estat. } & \multicolumn{12}{|c|}{ Alturas relativas de medição (\%) } \\
\hline & & $\mathbf{0}$ & 5 & 15 & 25 & 35 & 45 & 55 & 65 & 75 & 85 & 95 & 100 \\
\hline \multirow{4}{*}{$5^{\circ} \mathrm{Grau}$} & $\mathrm{D}$ & 0,273 & $-0,844$ & 0,395 & 0,188 & $-0,012$ & $-0,084$ & $-0,223$ & $-0,007$ & 0,179 & $-0,091$ & $-0,454$ & 0,192 \\
\hline & SQRR & 1,139 & 0,267 & 0,146 & 0,142 & 0,183 & 0,237 & 0,469 & 0,625 & 0,799 & 1,394 & 2,189 & 3,172 \\
\hline & SD & 21,642 & 19,046 & 16,557 & 15,851 & 15,549 & 15,002 & 14,082 & 12,971 & 11,953 & 11,203 & 10,579 & 10,131 \\
\hline & $\mathrm{RP}$ & 1,965 & $-4,304$ & 2,531 & 1,149 & $-0,611$ & $-0,878$ & $-1,278$ & 0,642 & 2,164 & 0,049 & $-3,590$ & 2,302 \\
\hline \multirow{4}{*}{$\begin{array}{l}\text { Hradetzky } \\
(1976)\end{array}$} & $\mathrm{D}$ & $-0,133$ & $-0,059$ & $-0,013$ & $-0,070$ & 0,141 & 0,209 & $-0,122$ & $-0,198$ & $-0,080$ & $-0,022$ & $-0,122$ & $-0,023$ \\
\hline & SQRR & 1,075 & 0,181 & 0,105 & 0,131 & 0,186 & 0,250 & 0,469 & 0,608 & 0,739 & 1,415 & 2,265 & 3,008 \\
\hline & SD & 22,068 & 18,222 & 16,984 & 16,121 & 15,388 & 14,695 & 13,976 & 13,171 & 12,224 & 11,130 & 10,230 & 10,356 \\
\hline & $\mathrm{RP}$ & 0,000 & 0,021 & $-0,052$ & $-0,545$ & 0,426 & 1,193 & $-0,529$ & $-0,887$ & $-0,105$ & 0,704 & $-0,304$ & 0,080 \\
\hline \multirow{5}{*}{$\begin{array}{l}\text { Kozak et } \\
\text { al. (1969) }\end{array}$} & $\mathrm{D}$ & 1,517 & $-1,386$ & $-1,177$ & $-0,771$ & $-0,045$ & 0,482 & 0,468 & 0,480 & 0,371 & $-0,155$ & $-0,896$ & $-0,665$ \\
\hline & SQRR & 1,663 & 0,431 & 0,342 & 0,250 & 0,184 & 0,308 & 0,603 & 0,800 & 0,884 & 1,379 & 2,264 & 2,852 \\
\hline & SD & 20,338 & 19,614 & 18,204 & 16,856 & 15,584 & 14,409 & 13,357 & 12,460 & 11,752 & 11,270 & 11,042 & 11,029 \\
\hline & $\mathrm{RP}$ & 8,504 & $-7,076$ & $-6,751$ & $-4,880$ & $-0,832$ & 3,204 & 4,079 & 4,770 & 3,910 & $-0,540$ & $-7,631$ & $-6,032$ \\
\hline & \multicolumn{13}{|c|}{ Notas } \\
\hline \multicolumn{2}{|l|}{$5^{\circ} \mathrm{Grau}$} & 7 & 8 & 7 & 7 & 6 & 6 & 9 & 6 & 8 & 7 & 7 & 8 \\
\hline \multicolumn{2}{|c|}{ Hradetzky (1976) } & 7 & 4 & 5 & 5 & 8 & 8 & 5 & 8 & 6 & 8 & 6 & 6 \\
\hline \multicolumn{2}{|c|}{ Kozak et al. (1969) } & 10 & 12 & 12 & 12 & 10 & 10 & 10 & 10 & 10 & 9 & 11 & 10 \\
\hline
\end{tabular}


O modelo de Kozak foi o que mostrou o pior desempenho, tendo pontuação nas classes de 6 a 9 anos, 13 a 15 anos e 16 a 19 anos, com $8 \%, 8 \%$ e 4\%, respectivamente.

Na figura 2, mostram-se os comportamentos do perfil ao longo do fuste para as classes de idade e para o conjunto total dos dados. Para representar o perfil de cada classe e do conjunto total dos dados, foi escolhido o modelo de melhor acurácia, conforme a tabela 7 .

Tabela 7. Classificação dos modelos nas posições do fuste por classe de idade e para o conjunto total dos dados, e valores percentuais das vezes em que o modelo ocupou a primeira posição para a estimativa dos diâmetros.

Table 7. Ranking of the fitted equations at the stem positions by age class and by the whole data set (total) for diameter estimates at every position along the tree stem for the total data set.

\begin{tabular}{cccccc}
\hline \multirow{2}{*}{ Altura (\%) } & \multicolumn{5}{c}{ Classes de idade (anos) } \\
\cline { 2 - 6 } & $\mathbf{6 - 9}$ & $\mathbf{1 0} \mathbf{- 1 2}$ & $\mathbf{1 3} \mathbf{- 1 5}$ & $\mathbf{1 6} \mathbf{- 1 9}$ & Total \\
\hline 0 & $\mathrm{Q}-\mathrm{H}$ & $\mathrm{Q}-\mathrm{H}$ & $\mathrm{H}$ & $\mathrm{Q}$ & $\mathrm{H}$ \\
5 & $\mathrm{H}$ & $\mathrm{H}$ & $\mathrm{H}$ & $\mathrm{H}$ & $\mathrm{H}$ \\
15 & $\mathrm{Q}-\mathrm{H}$ & $\mathrm{H}$ & $\mathrm{H}$ & $\mathrm{H}$ & $\mathrm{H}$ \\
25 & $\mathrm{Q}-\mathrm{H}$ & $\mathrm{H}$ & $\mathrm{H}$ & $\mathrm{H}$ & $\mathrm{H}$ \\
35 & $\mathrm{Q}$ & $\mathrm{Q}$ & $\mathrm{Q}$ & $\mathrm{Q}$ & $\mathrm{Q}$ \\
45 & $\mathrm{H}-\mathrm{K}$ & $\mathrm{Q}$ & $\mathrm{K}$ & $\mathrm{H}$ & $\mathrm{H}$ \\
55 & $\mathrm{Q}-\mathrm{H}$ & $\mathrm{H}$ & $\mathrm{H}$ & $\mathrm{H}$ & $\mathrm{H}$ \\
65 & $\mathrm{Q}-\mathrm{H}$ & $\mathrm{Q}$ & $\mathrm{Q}$ & $\mathrm{H}$ & $\mathrm{H}$ \\
75 & $\mathrm{Q}-\mathrm{H}$ & $\mathrm{H}$ & $\mathrm{H}$ & $\mathrm{H}$ & $\mathrm{H}$ \\
85 & $\mathrm{Q}-\mathrm{K}$ & $\mathrm{Q}$ & $\mathrm{Q}$ & $\mathrm{Q}$ & $\mathrm{H}$ \\
95 & $\mathrm{Q}$ & $\mathrm{H}$ & $\mathrm{Q}$ & $\mathrm{Q}-\mathrm{K}$ & $\mathrm{Q}-\mathrm{H}$ \\
100 & $\mathrm{Q}$ & $\mathrm{H}$ & $\mathrm{H}$ & $\mathrm{Q}$ & $\mathrm{H}$ \\
\hline $\mathrm{Q}$ & $54 \%$ & $38 \%$ & $34 \%$ & $38 \%$ & $12 \%$ \\
$\mathrm{H}$ & $38 \%$ & $62 \%$ & $58 \%$ & $58 \%$ & $88 \%$ \\
$\mathrm{~K}$ & $8 \%$ & $0 \%$ & $8 \%$ & $4 \%$ & $0 \%$ \\
Modelos: Q $=$ Polinômio de Quinto Grau; H $=$ Potências Fracionárias (HRADETZKY, 1976); K = Kozak et al. (1969)
\end{tabular}

Em geral, nas curvas das classes de idade ocorreu uma tendência pouco definida no perfil ao longo do fuste. Com o aumento da idade, houve uma tendência de melhorar a forma do fuste, ou seja, diminuir o afilamento. Já na porção inferior do fuste ocorre o contrário: fica mais irregular, principalmente até a primeira medição ( $10 \%$ da altura total do fuste).

A figura 3 mostra os perfis médios reais e estimados para o conjunto total dos dados (não agrupados) para os três modelos polinomiais estudados. Os demais perfis por classe de idade também foram feitos, e na maioria dos casos apresentaram comportamentos semelhantes.

Conforme a figura 3, o modelo de Potências Fracionárias foi o que propiciou melhor estimativa do perfil ao longo de todas as alturas relativas. Já os modelos de $5^{\circ}$ Grau e o de Kozak apresentaram tendenciosidade, ora subestimando ora superestimando. $\mathrm{O}$ modelo de $5^{\circ}$ Grau, principalmente até a posição de $15 \%$ da altura relativa do fuste, propiciou uma superestimativa. Nas demais posições, as variações são quase imperceptíveis. O modelo de Kozak mostra uma perceptível tendenciosidade até a altura relativa de $45 \%$, com uma superestimativa, depois de 45 a $85 \%$ uma subestimativa e posteriormente, até $100 \%$, novamente uma superestimativa. Essas tendenciosidades do modelo de Kozak ocorreram na maioria das classes de idade.

Em geral, para todas as classes de idade, os modelos de $5^{\circ}$ Grau e o de Potências Fracionárias apresentaram estimativas mais acuradas, com alguma tendenciosidade, ora perceptível, ora pouco perceptível. Já o modelo de Kozak, em quase todas as classes, apresentou fortes tendenciosidades ao longo das posições relativas do fuste. 


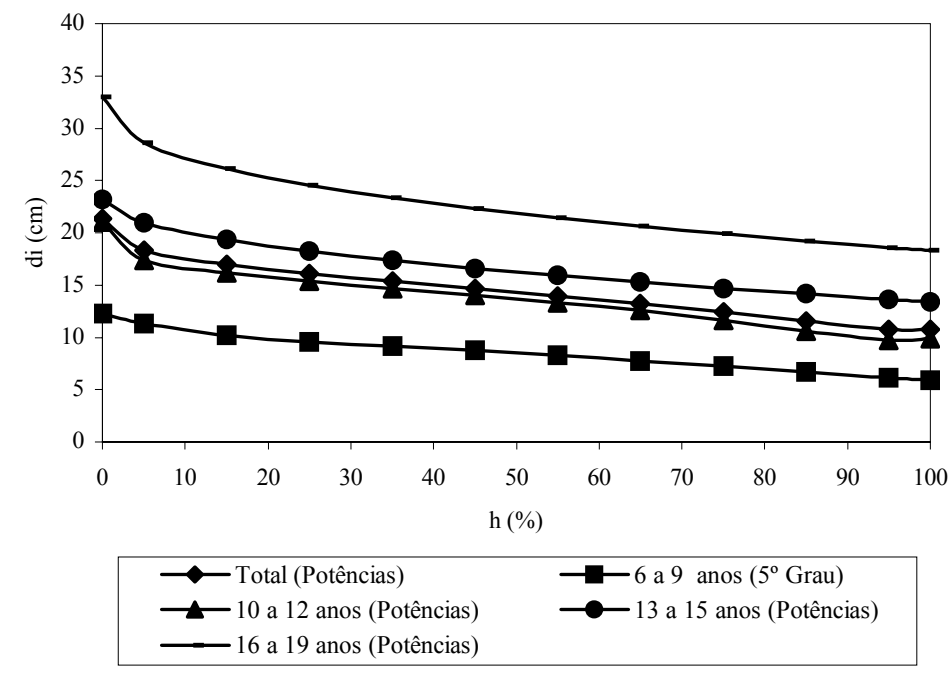

Figura 2. Perfil médio dos fustes para cada classe de idade e para o conjunto total dos dados. Figure 2. Average stem profile for each age class and for the total data set.
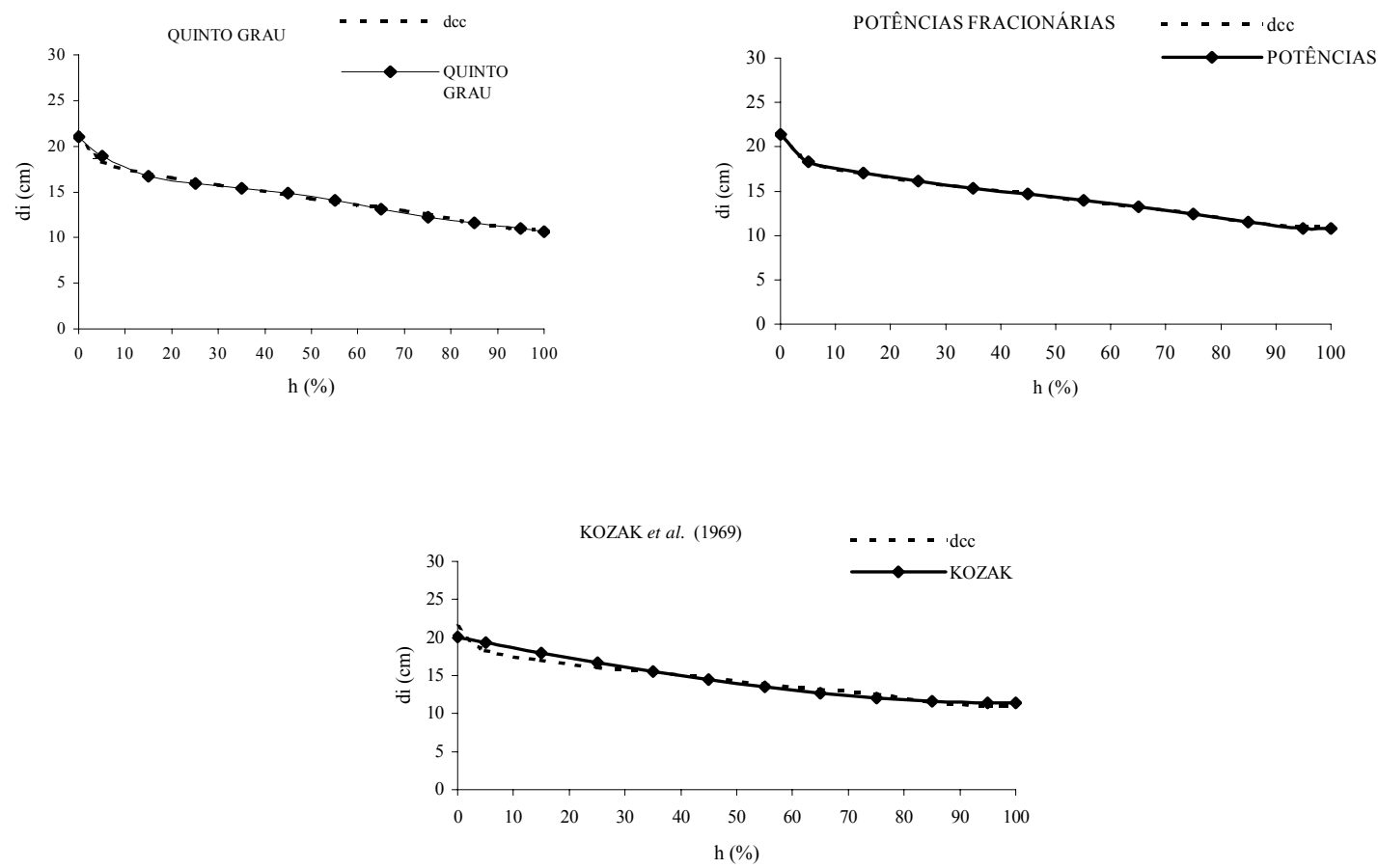

Figura 3. Perfis médios reais e estimados pelos modelos polinomiais não-segmentados para o conjunto total dos dados para os três modelos ajustados.

Figure 3. Observed average stem profiles and the estimated one by the three fitted taper equations for the total data set. 


\section{Acuracidade dos modelos não-segmentados na estimativa dos volumes}

A figura 4 mostra a análise gráfica dos resíduos percentuais em função do volume estimado $\left(\mathrm{m}^{3}\right)$ para o conjunto total dos dados (não agrupados). Esses gráficos também foram feitos para cada classe de idade. Pode-se observar alguma tendenciosidade na estimativa dos diâmetros e também em todas classes de idade. Nem sempre a equação de afilamento que apresentou a melhor pontuação, conforme será mostrado a seguir, deixa de apresentar tendenciosidade quando usada para estimativa do volume ao longo da linha média, indicando que a pontuação não pode ser usada como único requisito na escolha de um modelo.

Como mostrado na figura 4, em geral todos os modelos apresentaram alguma tendenciosidade ao longo da linha média. Esse mesmo comportamento verificou-se nas diferentes classes de idade.
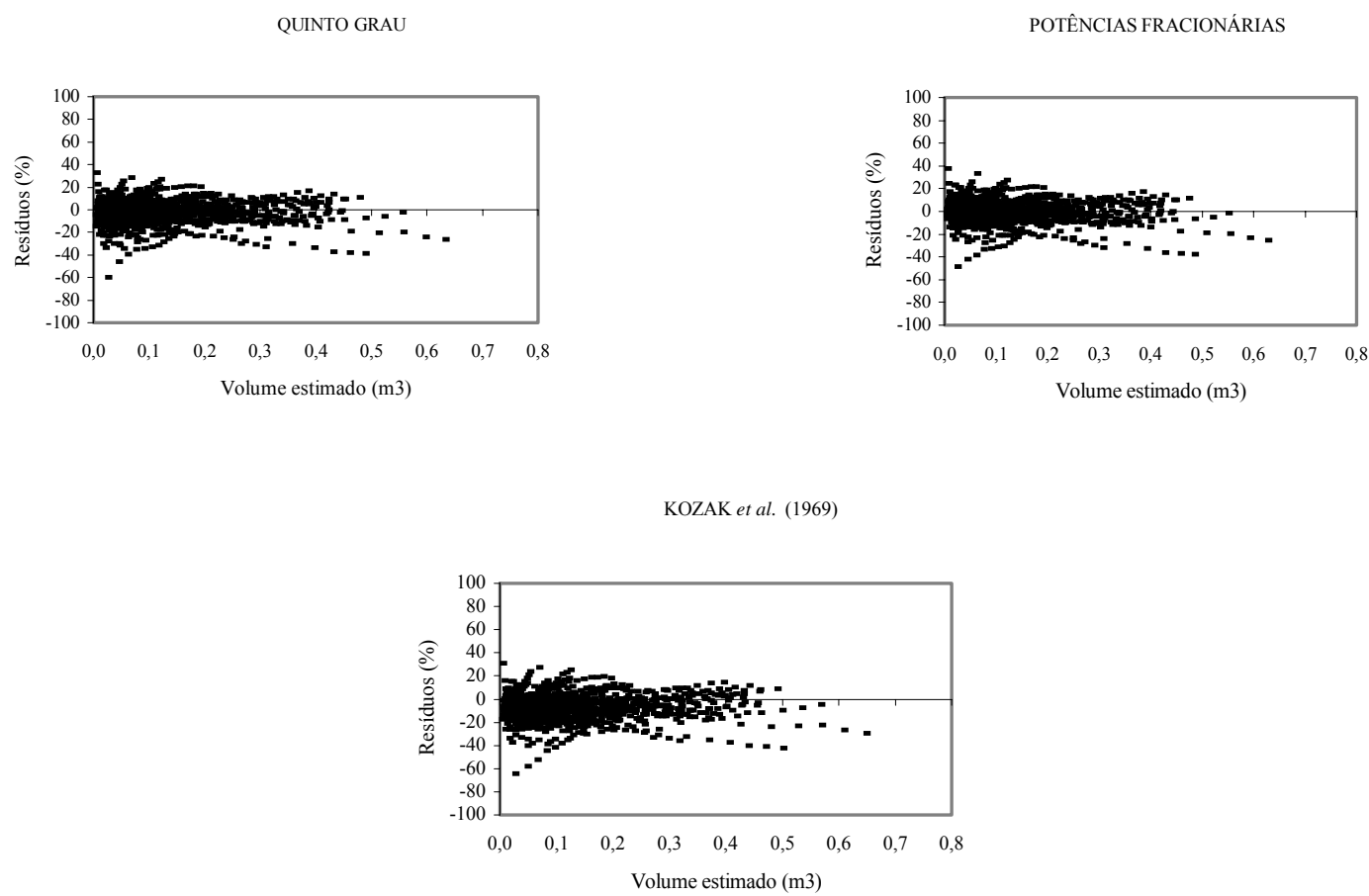

Figura 4. Distribuição gráfica dos resíduos em percentagem em função do volume estimado para o conjunto total dos dados para os três modelos ajustados.

Figure 4. Graphic residual distribution in percentage over estimated volume for the total data set for the three fitted equations.

A tabela 8 apresenta as estatísticas Desvio (D), Soma de Quadrados dos Resíduos Relativos (SQRR), Desvio Padrão das Diferenças (SD) e Resíduo Percentual (RP). Essa tabela apresenta apenas os resultados obtidos pelos três modelos polinomiais não-segmentados para o ajuste do total dos dados (não agrupados) para a variável volume em cada altura relativa do fuste, onde os volumes são acumulativos. Exemplificando: os volumes são calculados de 0 até $10 \%$ da altura relativa, de 0 a $20 \%$ e assim até totalizar o volume total ( 0 a $100 \%)$. Tabelas desse tipo também foram feitas para cada classe de idade, como mostra a tabela 9, para a classe de 10 a 12 anos.

Em geral, os valores da estatística Desvio (D) foram próximos de zero para a maioria dos casos. O modelo de Hradetzky foi o que apresentou os menores valores no somatório na maioria das posições, principalmente nas posições inferiores do fuste (até a altura de $60 \%$ ), sendo, portanto, o modelo mais acurado para a estimativa do volume para o conjunto total dos dados (Tabela 8). 
A partir dessas estatísticas, foi feita uma classificação dos modelos baseada na acurácia para estimar os volumes parciais acumulados nas diferentes posições do fuste. O modelo que obteve as menores notas foi considerado o mais acurado. Quando dois modelos foram selecionados em uma mesma posição, é porque apresentaram o mesmo número de pontos no processo de classificação. Quando se calcula a percentagem no caso dos modelos empatados, considera-se o peso de 0,5 para cada um, de modo que o somatório seja de 100\% (Tabela 10).

Tabela 8. Estatísticas dos modelos polinomiais não-segmentados para a estimativa dos volumes ao longo do fuste das árvores de Mimosa scabrella, para o ajuste do conjunto total dos dados (não agrupados).

Table 8. Statistics of the fitted taper equations for volume estimate for the total data set.

\begin{tabular}{|c|c|c|c|c|c|c|c|c|c|c|c|}
\hline \multirow{2}{*}{ Modelo } & \multirow[b]{2}{*}{ Estat. } & \multicolumn{10}{|c|}{ Alturas relativas de medição (\%) } \\
\hline & & 10 & 20 & 30 & 40 & 50 & 60 & 70 & 80 & 90 & 100 \\
\hline \multirow{4}{*}{$5^{\circ} \mathrm{Grau}$} & $\mathrm{D}$ & $-0,003$ & $-0,002$ & $-0,002$ & $-0,002$ & $-0,002$ & $-0,003$ & $-0,002$ & $-0,002$ & $-0,002$ & $-0,002$ \\
\hline & SQRR & 2,271 & 0,952 & 0,737 & 0,729 & 0,793 & 0,894 & 1,053 & 1,216 & 1,406 & 1,721 \\
\hline & SD & 0,040 & 0,070 & 0,098 & 0,124 & 0,148 & 0,170 & 0,189 & 0,205 & 0,220 & 0,233 \\
\hline & $\mathrm{RP}$ & $-7,048$ & $-2,602$ & $-1,107$ & $-0,802$ & $-0,840$ & $-0,848$ & $-0,458$ & 0,011 & 0,080 & 0,140 \\
\hline \multirow{4}{*}{$\begin{array}{l}\text { Hradetzky } \\
(1976)\end{array}$} & $\mathrm{D}$ & 0,000 & $-0,001$ & $-0,001$ & $-0,001$ & 0,000 & $-0,001$ & $-0,001$ & 0,000 & 0,000 & 0,000 \\
\hline & SQRR & 1,931 & 0,917 & 0,739 & 0,738 & 0,808 & 0,911 & 1,076 & 1,239 & 1,434 & 1,761 \\
\hline & SD & 0,037 & 0,069 & 0,097 & 0,123 & 0,146 & 0,168 & 0,187 & 0,204 & 0,218 & 0,231 \\
\hline & $\mathrm{RP}$ & $-0,174$ & $-0,047$ & $-0,036$ & 0,243 & 0,464 & 0,445 & 0,543 & 0,724 & 0,802 & 0,984 \\
\hline \multirow{5}{*}{$\begin{array}{l}\text { Kozak et } \\
\text { al. (1969) }\end{array}$} & $\mathrm{D}$ & $-0,003$ & $-0,007$ & $-0,009$ & $-0,009$ & $-0,008$ & $-0,008$ & $-0,006$ & $-0,005$ & $-0,005$ & $-0,006$ \\
\hline & SQRR & 2,682 & 1,943 & 1,636 & 1,334 & 1,129 & 1,058 & 1,109 & 1,219 & 1,396 & 1,701 \\
\hline & SD & 0,041 & 0,076 & 0,107 & 0,133 & 0,156 & 0,176 & 0,194 & 0,210 & 0,224 & 0,239 \\
\hline & $\mathrm{RP}$ & $-9,507$ & $-10,001$ & $-9,259$ & $-7,686$ & $-5,930$ & $-4,425$ & $-3,085$ & $-2,151$ & $-1,991$ & $-2,274$ \\
\hline & \multicolumn{11}{|c|}{ Notas } \\
\hline \multicolumn{2}{|l|}{$5^{\circ} \mathrm{Grau}$} & 8 & 8 & 8 & 8 & 7 & 7 & 6 & 6 & 7 & 7 \\
\hline \multicolumn{2}{|c|}{ Hradetzky (1976) } & 4 & 4 & 4 & 4 & 5 & 5 & 6 & 7 & 7 & 7 \\
\hline \multicolumn{2}{|c|}{ Kozak et al. (1969) } & 12 & 12 & 12 & 12 & 12 & 12 & 12 & 11 & 10 & 10 \\
\hline
\end{tabular}

Sendo: $\mathrm{D}$ = desvio; $\mathrm{SQRR}=$ soma de quadrados dos resíduos relativos; $\mathrm{SD}$ = desvio padrão das diferenças; $\mathrm{RP}$ = resíduo percentual.

Tabela 9. Estatísticas dos modelos polinomiais não-segmentados para a estimativa dos volumes ao longo do fuste das árvores de Mimosa scabrella, na classe de idade 10 a 12 anos.

Table 9. Statistics of the fitted taper equations for volume estimates for the age class 10 to 12 years old.

\begin{tabular}{|c|c|c|c|c|c|c|c|c|c|c|c|}
\hline \multirow[b]{2}{*}{ Modelo } & \multirow[b]{2}{*}{ Estat. } & \multicolumn{10}{|c|}{ Alturas relativas de medição (\%) } \\
\hline & & 10 & 20 & 30 & 40 & 50 & 60 & 70 & 80 & 90 & 100 \\
\hline \multirow{4}{*}{$5^{\circ} \mathrm{Grau}$} & $\mathrm{D}$ & $-0,003$ & $-0,003$ & $-0,002$ & $-0,002$ & $-0,002$ & $-0,003$ & $-0,003$ & $-0,003$ & $-0,003$ & $-0,004$ \\
\hline & SQRR & 1,144 & 0,432 & 0,352 & 0,343 & 0,374 & 0,422 & 0,469 & 0,525 & 0,622 & 0,751 \\
\hline & $\mathrm{SD}$ & 0,034 & 0,059 & 0,082 & 0,104 & 0,124 & 0,142 & 0,158 & 0,171 & 0,182 & 0,192 \\
\hline & $\mathrm{RP}$ & $-10,069$ & $-3,902$ & $-2,149$ & $-1,851$ & $-1,731$ & $-1,720$ & $-1,323$ & $-0,806$ & $-0,600$ & $-0,719$ \\
\hline \multirow{4}{*}{$\begin{array}{l}\text { Hradetzky } \\
\text { (1976) }\end{array}$} & $\mathrm{D}$ & $-0,001$ & $-0,001$ & $-0,001$ & $-0,001$ & 0,000 & $-0,001$ & $-0,001$ & $-0,001$ & $-0,001$ & $-0,002$ \\
\hline & SQRR & 0,716 & 0,375 & 0,339 & 0,335 & 0,373 & 0,422 & 0,474 & 0,533 & 0,634 & 0,768 \\
\hline & SD & 0,031 & 0,057 & 0,081 & 0,102 & 0,122 & 0,140 & 0,155 & 0,169 & 0,180 & 0,190 \\
\hline & $\mathrm{RP}$ & $-0,775$ & $-0,464$ & $-0,603$ & $-0,232$ & 0,265 & 0,207 & 0,131 & 0,209 & 0,436 & 0,510 \\
\hline \multirow{4}{*}{$\begin{array}{l}\text { Kozak et } \\
\text { al. (1969) }\end{array}$} & $\mathrm{D}$ & $-0,005$ & $-0,008$ & $-0,010$ & $-0,010$ & $-0,009$ & $-0,008$ & $-0,007$ & $-0,007$ & $-0,007$ & $-0,009$ \\
\hline & SQRR & 1,537 & 1,243 & 1,071 & 0,826 & 0,635 & 0,557 & 0,535 & 0,557 & 0,642 & 0,775 \\
\hline & SD & 0,035 & 0,065 & 0,091 & 0,113 & 0,132 & 0,149 & 0,163 & 0,175 & 0,187 & 0,198 \\
\hline & $\mathrm{RP}$ & $-13,425$ & $-13,203$ & $-12,119$ & $-10,030$ & $-7,615$ & $-5,784$ & $-4,339$ & $-3,363$ & $-3,079$ & $-3,542$ \\
\hline \multicolumn{12}{|c|}{ Notas } \\
\hline \multicolumn{2}{|l|}{$5^{\circ} \mathrm{Grau}$} & 8 & 8 & 8 & 8 & 8 & 7 & 7 & 7 & 7 & 7 \\
\hline \multicolumn{2}{|c|}{ Hradetzky (1976) } & 4 & 4 & 4 & 4 & 4 & 5 & 5 & 5 & 5 & 5 \\
\hline \multicolumn{2}{|c|}{ Kozak et al. (1969) } & 12 & 12 & 12 & 12 & 12 & 12 & 12 & 12 & 12 & 12 \\
\hline
\end{tabular}


Para o conjunto total de árvores, o modelo de Potências Fracionárias foi o que estimou os volumes com maior acuracidade, representando $75 \%$ das posições, seguido pelo modelo de $5^{\circ} \mathrm{Grau}$, com $25 \%$. Na tabela 10, fica evidente a predominância do modelo de Potências Fracionárias ao longo de todo o fuste, exceto apenas na classe de 6 a 9 anos, na qual o modelo de $5^{\circ}$ Grau foi o que ocupou o primeiro lugar, com $90 \%$. Há um destaque para o modelo de Potências Fracionárias na classe de 10 a 12 anos, em que representou $100 \%$ das posições.

Na classe de idade de 13 a 15 anos (Tabela 10), houve empate do modelo de Potências Fracionárias com o de $5^{\circ} \mathrm{Grau}$, cada um representando $50 \%$ das posições.

O modelo de Kozak foi o que apresentou o pior resultado, pontuando apenas na classe de 6 a 9 anos, com $10 \%$ das posições.

Tabela 10.Classificação dos modelos nas posições do fuste por classe de idade e para o conjunto total dos dados e valores percentuais para as vezes em que o modelo ocupou a primeira posição para estimativa dos volumes totais e parciais.

Table 10. Ranking of the fitted equations at the stem positions by age class and by the whole data set (total) for volume estimate at every position along the tree stem for the total data set.

\begin{tabular}{cccccc}
\hline \multirow{2}{*}{ Altura (\%) } & \multicolumn{5}{c}{ Classes de idade (anos) } \\
\cline { 2 - 6 } & $\mathbf{6 - 9}$ & $\mathbf{1 0 - 1 2}$ & $\mathbf{1 3 - 1 5}$ & $\mathbf{1 6 - 1 9}$ & TOTAL \\
\hline 10 & $\mathrm{Q}$ & $\mathrm{H}$ & $\mathrm{H}$ & $\mathrm{H}$ & $\mathrm{H}$ \\
20 & $\mathrm{Q}$ & $\mathrm{H}$ & $\mathrm{Q}$ & $\mathrm{H}$ & $\mathrm{H}$ \\
30 & $\mathrm{Q}$ & $\mathrm{H}$ & $\mathrm{Q}$ & $\mathrm{H}$ & $\mathrm{H}$ \\
40 & $\mathrm{Q}$ & $\mathrm{H}$ & $\mathrm{Q}$ & $\mathrm{Q}-\mathrm{H}$ & $\mathrm{H}$ \\
50 & $\mathrm{Q}$ & $\mathrm{H}$ & $\mathrm{Q}$ & $\mathrm{Q}-\mathrm{H}$ & $\mathrm{H}$ \\
60 & $\mathrm{Q}$ & $\mathrm{H}$ & $\mathrm{H}$ & $\mathrm{Q}-\mathrm{H}$ & $\mathrm{H}$ \\
70 & $\mathrm{Q}$ & $\mathrm{H}$ & $\mathrm{H}$ & $\mathrm{Q}$ & $\mathrm{Q}-\mathrm{H}$ \\
80 & $\mathrm{Q}$ & $\mathrm{H}$ & $\mathrm{Q}-\mathrm{H}$ & $\mathrm{Q}-\mathrm{H}$ & $\mathrm{Q}$ \\
90 & $\mathrm{Q}-\mathrm{K}$ & $\mathrm{H}$ & $\mathrm{Q}-\mathrm{H}$ & $\mathrm{Q}-\mathrm{H}$ & $\mathrm{Q}-\mathrm{H}$ \\
100 & $\mathrm{Q}-\mathrm{K}$ & $\mathrm{H}$ & $\mathrm{H}$ & $\mathrm{Q}-\mathrm{H}$ & $\mathrm{Q}-\mathrm{H}$ \\
\hline $\mathrm{Q}$ & $90 \%$ & $0 \%$ & $50 \%$ & $40 \%$ & $25 \%$ \\
$\mathrm{H}$ & $0 \%$ & $100 \%$ & $50 \%$ & $60 \%$ & $75 \%$ \\
$\mathrm{~K}$ & $10 \%$ & $0 \%$ & $0 \%$ & $0 \%$ & $0 \%$ \\
\hline Modelos: Q $=$ Polinômio de Quinto Grau; H $=$ Potências Fracionárias (HRADETZKY, 1976); K = Kozak et al. (1969)
\end{tabular}

A figura 5 mostra os volumes médios reais e estimados para o conjunto total dos dados (não agrupados) para os três modelos polinomiais estudados. Os demais perfis para cada classe de idade também foram feitos. O modelo de Potências e o de $5^{\circ}$ Grau foram os que apresentaram melhores estimativas do volume ao longo de todas as alturas relativas, com alguma tendenciosidade pouco perceptível em alguns pontos do fuste, ora subestimando, ora superestimando. O modelo de Kozak et al. (1969) superestima sistematicamente os volumes ao longo de todas as posições relativas do fuste, não sendo, portanto, recomendado para esse fim.

Em todos as classes de idade verifica-se a alta eficiência dos modelos de Potências e de $5^{\circ} \mathrm{Grau}$ em estimativa de volume. O modelo de Kozak et al. (1969) mostra baixa acurácia na estimativa dos volumes parciais em todas as classes.

\section{Validação das equações de afilamento utilizadas para a estimativa de volumes}

Na tabela 11, estão apresentados os resultados dos $\chi^{2}$ calculados para o nível de probabilidade de $95 \%$, bem como os graus de liberdade para cada classe de idade e para o conjunto total. Os valores tabelados foram comparados com os calculados. Se o $\chi^{2}$ Calculado for menor que o $\chi^{2}$ tabelado, aceita-se a hipótese de que o modelo de afilamento usado para estimativa em cada classe de idade, e para o conjunto total dos dados, é acurado. Para a classe de idade de 13 a 15 anos, houve empate entre os modelos de Potências Fracionárias e o de $5^{\circ}$ Grau, motivo pelo qual se fez a validação para ambos. 

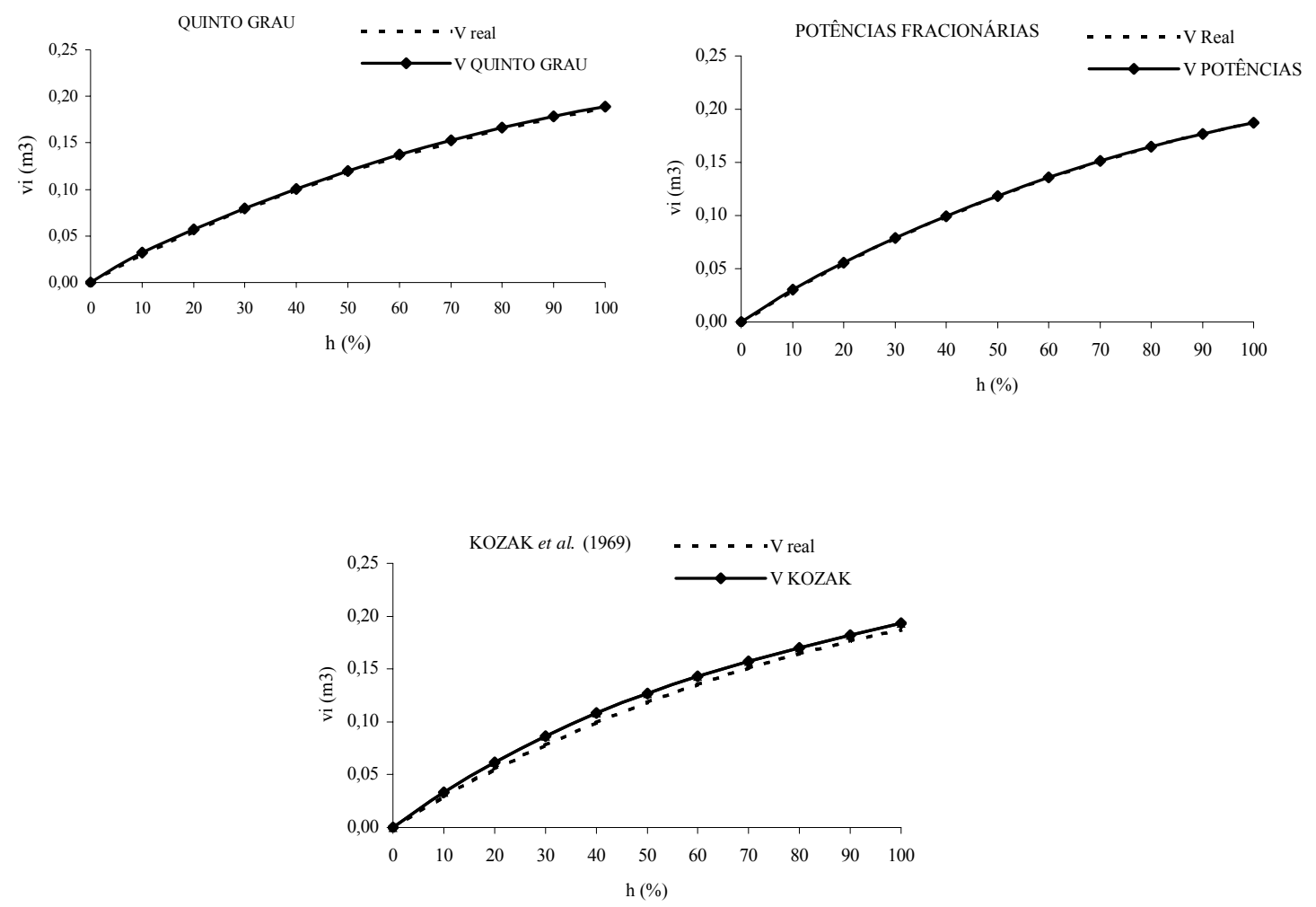

Figura 5. Volumes reais e estimados pelos modelos polinomiais não-segmentados para o conjunto total dos dados para os três modelos ajustados.

Figure 5. Observed and estimated volumes by the tree fitted taper equations for the total data set.

Os $\chi^{2}$ calculados foram menores que os $\chi^{2}$ tabelados para os níveis de probabilidade de $95 \%$. Portanto, todos os modelos validados podem ser usados na estimativa dos volumes nas respectivas classes de idade e para o conjunto total dos dados (Tabela 11). Nessa tabela, são mostrados o erro padrão da estimativa em porcentagem (Syx\%) para a estimativa do volume, para o conjunto total dos dados e para cada classe de idade. Esses erros foram calculados a partir da estimativa das árvores que foram xilometradas, utilizando-se para isso a equação de melhor acurácia para cada classe. Depois, esses volumes estimados foram comparados com os respectivos volumes calculados pela técnica do deslocamento de água (xilômetro). Os resultados mostraram valores consideravelmente altos em quase todas as classes, exceto apenas na classe de 10 a 12 anos, em que o erro padrão da estimativa foi de $14,04 \%$. Esses erros também foram altos para o conjunto total dos dados.

Tabela 11. Valores de $\chi^{2}$ calculados para cada classe de idade e para o conjunto total dos dados.

Table 11. $\chi^{2}$ calculated values for every age class and for the total data set.

\begin{tabular}{cccccc}
\hline $\begin{array}{c}\text { Classe de idade } \\
\text { (anos) }\end{array}$ & $\begin{array}{c}\mathbf{N}^{\mathbf{0}} \text { árvores } \\
\text { xilometradas }\end{array}$ & $\begin{array}{c}\text { Graus de } \\
\text { liberdade }\end{array}$ & $\chi^{2}$ Calculado & Teste & Syx \%( $\left.\hat{\mathbf{V}}_{\mathrm{i}}\right)$ \\
\hline $6-9$ & 22 & 21 & 0,1289738 & $\mathrm{NS}$ & 48,38 \\
$10-12$ & 23 & 22 & 0,0446757 & $\mathrm{NS}$ & 14,04 \\
$13-15$ & 5 & 4 & 0,018918 & $\mathrm{NS}$ & 31,81 \\
$13-15$ & 5 & 4 & 0,018665 & $\mathrm{NS}$ & 31,43 \\
$16-19$ & 3 & 2 & 0,0199322 & $\mathrm{NS}$ & 26,29 \\
\hline Total & 53 & 52 & 0,2118596 & $\mathrm{NS}$ & 21,12 \\
\hline NS = Não significativo ao nível de 5\%. & & & &
\end{tabular}




\section{CONCLUSÕES}

- A equação de Potências Fracionárias é a mais acurada para estimativa dos diâmetros e volumes em quase todas classes de idade, e a melhor para o conjunto total dos dados.

- O modelo de Kozak et al. (1969) apresentou tendenciosidade ao longo do fuste, tanto na estimativa do diâmetro como do volume, superestimando ou subestimando-o em todas as classes e no conjunto total dos dados. Portanto, não deve ser utilizado nas estimativas.

- O modelo de Potências Fracionárias mostrou uma alta eficiência na estimativa dos diâmetros, principalmente nas partes inferiores do fuste.

- A forma do fuste é influenciada pelo aumento da idade, diminuindo o afilamento.

- A estimativa do volume pela equação de afilamento, quando comparado com seu respectivo volume calculado pela técnica de deslocamento de água (xilômetro), apresentou alto erro padrão da estimativa (Syx\%) em quase todas as classes de idade, bem como para o conjunto total dos dados.

- Todos os modelos que foram validados na estimativa do volume são recomendados na utilização para esse fim.

\section{REFERÊNCIAS}

AHRENS, S.; HOLBERT, D. Uma função para forma de tronco e volume de Pinus taeda L. Boletim de Pesquisa Florestal, Curitiba, n. 3,.p.37-68, 1981.

ASSIS, A. L. de. Avaliação de modelos polinomiais segmentados e não-segmentados na estimativa de diâmetros e volumes comerciais de Pinus taeda. 189p. Dissertação (Mestrado em Engenharia Florestal) - Universidade Federal de Lavras, Lavras, 2000.

BARTOSZECK, A. C. de. P. e. S. Evolução da relação hipsométrica e da distribuição diamétrica em função dos fatores idade, sítio e densidade inicial em bracatingais da Região Metropolitana de Curitiba. 214f. Dissertação (Mestrado em Engenharia Florestal) - Setor de Ciências Agrárias, Universidade Federal do Paraná, Curitiba, 2000.

CARPANEZZI, A. et al. Manual técnico da bracatinga (Mimosa scabrella Bentham). Colombo: EMBRAPA-CNPF, 1988. (Documentos, 20).

FERREIRA, M. Z. Estudo de funções de afilamento para representar o perfil e o volume do fuste de Pinus taeda L. 196p. Dissertação (Mestrado em Engenharia Florestal) - Universidade Federal de Lavras, Lavras, 2004.

FERREIRA, S. O. Estudo da Forma do Fuste de Eucalyptus grandis e Eucalyptus cloeziana. 132p. Dissertação (Mestrado em Engenharia Florestal) - Universidade Federal de Lavras, Lavras, 1999.

FIGUEIREDO FILHO, A.; BORDERS, B. E.; HITCH, K. L. Taper equations for Pinus taeda plantations in southern Brazil. Forest Ecology and Management, Amsterdam, v. 83, p. 36-46, 1996.

HRADETZKY, J. Analyse und interpretation statistisher abhängigkeiten (Biometrische Beiträge zu aktuellen forschungsprojekten). Baden: Württemberg Mitteilungen der FVA, 1976. 146p. (Abt. Biometric und Informatik, 21).

IAPAR. Cartas climáticas do Estado do Paraná: 1994. Londrina, 1994.

KOZAK, A.; MUNRO, D. P.; SMITH, J. H. G. Taper functions and their application in forest inventory. Forest Chronicle, Toronto, v. 45, n. 4, p. 278-283, 1969.

MAACK, R. Geografia física do Estado do Paraná. 2 ed. Rio de Janeiro: J. Olympio, 1981. 450p.

MACHADO, S. do A.; NADOLNY, M. C. Comparação de métodos de cubagem de árvores e de diversos comprimentos de seção. In: CONGRESSO FLORESTAL E DO MEIO AMBIENTE DO PARANÁ, 3. 1991, Curitiba. Anais... Curitiba: Associação Paranaense de Engenheiros Florestais, 1991. p.89-104. 
PEDROSO. O; MATTOS, J. R. Estudos sobre madeiras no Rio Grande do Sul. Porto Alegre: IPRNR, 1987.

PRODAN, M. et al. Mensura Forestal. San José: IICA, 1997. 586p. (Investigacion y educacion en desarrolo sostenible, v. 1).

SCHOEPPER, W. Autimatisierung des Massen, Sorten and Wertberechnung stenender Waaldbestande Schriftenreihe Bad. [S.1.]: Wurtt-Forstl., 1966.

TONON, A. E. N. Efeitos da Densidade Inicial e do Sítio Sobre o Crescimento e a Produção de Bracatingais da Região Metropolitana de Curitiba. 193f. Dissertação (Mestrado em Engenharia Florestal) - Setor de Ciências Agrárias, Universidade Federal do Paraná, Curitiba. 1998. 